\title{
Some Studies on Digital Image Segmentation Techniques
}

\author{
K. Asish vardhan 1$)$
}

\begin{abstract}
The importance of this paper is to give a survey of advanced picture division methods. The issues of digital picture division speak to formidable difficulties for PC vision. The extensive variety of the issues of PC vision may make excellent utilisation of picture division. This paper examines and assesses the diverse strategies for division systems. We examine the fundamental propensity of every calculation with their applications, points of interest and burdens. This examination is helpful for deciding the appropriate utilisation of the picture division strategies and for enhancing their exactness and execution and furthermore for the primary goal, which planning new calculations.
\end{abstract}

Key words: Image Processing, Image Segmentation, Digital Images, Digital Image Processing, Segmentation Techniques

\section{Introduction}

Computerized picture preparing is essential to space for some reasons. Digital picture handling is a current subject in PC history. In the 1960s; Bell Labs and the University of Maryland, and a couple of different spots began to build up a few procedures for computerised picture handling. With application to satellite symbolism, wire photograph measures change medicinal imaging, videophone, character acknowledgement, and photograph improvement. The cost of preparing was genuinely high with the figuring hardware of that period. In the 1970s, picture preparing multiplied, when less expensive PCs and devoted equipment wound up accessible. Pictures could then be handled continuously, for some committed issues, for example, TV norms change. As broadly useful PCs turned out to be quicker, they began to take once again the part of committed equipment for everything except the most particular and register concentrated activities.

In computerised picture preparing, we utilise PC calculations to perform picture handling. In reality computerised picture handling has a few focal points over the simple picture preparing;

Received(January 03, 2019), Review Result(1st: January 21, 2019, 2nd: February 27, 2019), Accepted(March 10, 2019)

1) (Professor) Department of Computer Science \& Engineering, Vignan's Institute of Information Technology (A) Visakhapatnam, AP, India email: Satyasaikiran9@gmail.com 
first it gives a high number of calculations to be utilised with the information, second, we can stay away from some handling issues, for example, making a commotion and flag twisting amid flag preparing. In the 2000s, quick PCs ended up accessible for flag preparing, and computerised picture handling has turned into the mainstream type of picture handling. Hence, flag picture handling ended up flexible technique, and furthermore least expensive. Picture division is a critical part of numerous flag preparing procedure and its applications. The division methodology is to locate the better places of the shape indicates concurring the appearance data. Calculations in light of classifiers have generally been connected to fragment organs in medicinal pictures like cardiovascular and mind pictures. The objective of picture division process is parcelling the picture into districts. We can utilise division for picture pressure, question acknowledgement, and picture altering handling.

Computerized picture handling is gotten from the Greek word which implies secured composing and signifies "to stow away in the plain sight". As characterised by Cachin[1] Digital picture preparing is the artistry and art of conveying such that the nearness of a message cannot be recognised. Straightforward steganographic procedures have been used for many years, however with the expanding utilization of documents in computerized media new systems for data covering up have turned out to be required. This paper looks at some early cases of the Steganographic process and the general standards behind its utilisation. There will then be a dialogue of some particular procedures for concealing data in an assortment of configurations and the assaults that might be utilised to sidestep Digital picture preparing. Figure 1 indicates how data covering up can be separated into various territories. Computerized picture preparing can be utilised to shroud a message proposed for later recovery by a particular individual or gathering. For this situation, the point is to keep the message being identified by an outsider. Another real territory of Digital picture preparing is copyright stamping, where the message to be embedded is utilised to affirm copyright over an archive.

Picture preparing is a quickly developing region of software engineering. Its development had filled by innovative advances in computerised imaging, PC processors and mass stockpiling gadgets. Fields which customarily utilised simple imaging are currently changing to advanced frameworks, for their flexibility and reasonableness. Essential cases are medication, $1 \mathrm{~m}$ and video generation, photography, remote detecting, and security observing. These and different sources deliver tremendous volumes of advanced picture information consistently, more than would ever be inspected physically. Computerized picture preparing is concerned fundamentally with separating helpful data from pictures. Preferably, this is finished by PCs, with next to 
zero human mediation. Picture handling calculations might set at three levels.

At the most reduced level are those methods which bargain specifically with the crude, potentially uproarious pixel esteems, with denoising and edge location being great cases. In the centre are calculations which use low-level outcomes additionally implies, for example, division and edge connecting. At the most elevated amount are those techniques which endeavour to extricate semantic significance from the data gave by the lower levels, for instance, penmanship acknowledgement. The writing teems with calculations for accomplishing different picture handling errands. Be that as it may, there does not seem, by all accounts, to be any binding together rule managing a significant number of them. Some are one-dimensional flag preparing procedures which have been reached out to two measurements. Others apply techniques from elective orders to picture information in a to some degree unseemly way. Numerous are a similar essential calculation with parameter esteems changed to suit the current issue. On the other hand, the parameters improved as for a consistent preparing Image handling is a quickly developing territory of software engineering. Its development has been energised by mechanical advances in computerised imaging, PC processors and mass stockpiling gadgets.

\section{Related Work}

The issues of advanced picture division speak to formidable difficulties for PC vision. The extensive variety of the issues of PC vision may make excellent utilisation of picture division. Numerous scientists had made a few strategies to manage the issue of picture division. Zimmer et al.[2] made a strategy to recognise the portability of live cells utilising the dynamic shape (snakes) technique. Mukherjee et al.[3] adjusted a technique to deal with the following issue utilising limit strategy. Coskun et al.[4] utilised the opposite demonstrating to recognise the versatility of living cells. As of late, there have been various scientists has endeavoured to make a few picture division calculations as in (Krinidis et al., Mélange, et al., Mignotte et al.). In this paper, we figured out how to survey and condensed the real procedures for computerised picture division. We classified these procedures in light of the division strategy which the method is utilising. In Table 2.1 a correlation of the picture division techniques.

The writing flourishes with calculations for accomplishing different picture preparing assignments. Be that as it may, there does not have all the earmarks of being any bringing together standard directing a large number of them. Some are one-dimensional flag preparing methods which have been stretched out to two measurements. Others apply strategies from 
elective controls to picture information in a reasonably rude way. Numerous are a similar fundamental calculation with parameter esteems changed to suit the current issue. Then again, the parameters streamlined as for a reasonable preparing. Numerous current picture preparing calculations as of now make incomplete utilisation of the neighbourhood division idea. It is conceivable to look at these calculations regarding the neighbourhood division display they utilise. This makes their qualities and shortcomings more clear. Indeed, even well-known methods, for example, direct and rank _filters, can be surrounded regarding their use of neighbourhood division. By and large, the division is understood instead of express.

Hasty commotion is every now and again experienced in computerised picture transmission as a result of human-made clamour sources or disentangling blunders. Flag subordinate commotion is the photoelectron clamour of photograph detecting gadgets and the film-grain commotion of photographic movies[5]. Dot commotion that shows up in ultrasonic imaging and laser imaging is multiplicative clamour; i.e. it is subordinate flag commotion. Another illustration where straight methods fall flat is the situation of nonlinear picture debasements. Such debasements happen amid picture development and amid picture transmission through nonlinear channels[4][6]. The human visual recognition component has been appeared to have nonlinear qualities too[1][5].

Direct channels, which were initially utilized as a part of picture sifting applications, can't adapt to the nonlinearities of the picture arrangement display and can't consider the nonlinearities of human vision. Besides, human vision is exceptionally touchy to high-recurrence data. Picture edges and picture points of interest (e.g. corners and lines) have high-recurrence substance and convey critical data for visual discernment. Channels have a significant edge and picture detail safeguarding properties are exceedingly appropriate for advanced picture separating. A significant portion of the traditional straight computerised picture channels has low-pass qualities[7]. They tend to obscure edges and to obliterate lines, edges, and other excellent picture points of interest. These reasons have driven scientists to the utilisation of nonlinear separating methods. Nonlinear methods rose ahead of schedule in advanced picture handling. In any case, the more significant part of the related research had introduced in the previous decade. This exploration zone has had a dynamic advancement. The measure of research demonstrates this by and by distributed and the fame and across the board utilisation of nonlinear computerised handling in an assortment of uses. The more significant part of the presently available picture handling programming bundles incorporates nonlinear procedures (e.g. middle channels and morphological channels). A variety of nonlinear computerised picture preparing procedures has shown up in writing. 
One of the first confinements of nonlinear procedures to introduce is the absence of a binding together hypothesis that can include all current nonlinear channel classes. Each class of nonlinear preparing systems has its particular scientific apparatuses that can give sensibly great examination of its execution. Cross-treatment of these classes has been appeared to guarantee. For instance, scientific morphology and request measurement channels have too, the more significant part of the detailed work has been connected to advanced picture preparing. We should concentrate our introduction on electronic picture handling applications, keeping in mind the end goal to render it briefer. In the accompanying, we should centre on the portrayal of the request insights systems. Albeit such methods have been connected to advanced flag preparing. Since its first utilise, a few alterations and expansions of the middle channel have proposed. A significant number of them have strong hypothetical establishments from the hypothesis of vigorous insights[6][8]. Various chips for quick middle and $\mathrm{max} / \mathrm{min}$ separating have introduced in writing. The related endeavours for quick channel execution are checked on in this paper also[9].

\section{Problems in Dip}

\subsection{Edge Detection}

Edge location, an area part approach, creates an edge delineate contains vital data about the picture. The memory space required for capacity is moderately little, and the first picture can be reestablished effectively from its edge delineates. Numerous techniques have proposed for edge recognition in digital pictures proposed a strategy for edge-based picture division utilising Object Localization and Border Detection Criteria propose a strategy for edge discovery utilising Adaptive Neuro-Fuzzy System. The framework comprises of a MultiLayer Perceptron (MLP)like the system that performs picture division by versatile thresholding of the info picture proposed a technique for edge discovery utilising Fast Multilevel Fuzzy Edge Detection propose a strategy for edge location utilising wavelets for SEM pictures. This technique encourages nanoscale edge identification and portrayal by giving a practical edge assurance step.

Beamlets and multiscale picture investigation proposed a strategy for a productive FPGA usage of MRI picture separating and tumour portrayal utilising Xilinx System Generator[10] propose a technique for Architecture for sifting pictures utilising Xilinx System Generator proposed a strategy for Implementation and Evaluation of Image Processing Algorithms on Reconfigurable Architecture utilising C-based Hardware Descriptive Languages. Beamlet change 
and multiscale straight element extraction proposed. An outline of MRI Brain arrangement utilising FPGA execution proposed. FPGA execution of picture pressure utilising base up approach of Quadtree procedure proposed by [11][12].

\subsection{Signal to Error Ratio (SER)}

Removed logo picture and got the picture after both watermarking processes are broke down as far as the flag to blunder proportion. Impact of different scaling components can be seen on the estimations of a flag to mistake proportion (SER) for both got yield picture and removed logo picture. '.bmp' a picture to create watermark installed picture by embeddings a logo '.bmp.' a picture with non zero scaling factor.

Kinds of commotion: The past case represented the way in which a picture might be influenced by clamour amid the securing procedure. The properties of the commotion presented at each catch step are probably going to change. In any case, there are three standard clamour models which show well the sorts of commotion experienced in many pictures: added substance, multiplicative, and drive clamour.

\subsection{Added Substance Noise}

The Let $f^{\prime}(x, y)$ be the uproarious digitized rendition of the perfect picture $f(x, y)$ and $n(x, y$,$) / be a$ .clamor work. Which returns irregular qualities originating from a discretionary circulation added substance commotion is free of the pixel esteems in the first picture. Commonly $n(x, y)$ is symmetric around zero. This has the impact of not changing the normal brilliance of the picture or substantial parts thereof. Added substance clamour is a decent model for the warm commotion inside photograph electronic sensors.

\subsection{Multiplicative Commotion}

Multiplicative commotion, or dot clamour, is a subordinate flag type of commotion whose size identified with the estimation of the first pixel[13]. It portrays one straightforward frame it can take. However, a more mind-boggling capacity of the first-pixel esteem is additionally conceivable. Multiplicative commotion is estimation to the clamour experienced in pictures recorded on[10] and from manufactured opening radar. The past case delineated the way in which clamour might influence a picture amid the securing procedure. The properties of the commotion presented at each catch step are probably going to fluctuate. Be that as it may, 
there are three standard clamour models which demonstrate well the kinds of commotion experienced in many pictures: added substance, multiplicative, and motivation clamour.

\subsection{Drive Noise}

Drive commotion has the property of either leaving a pixel unmodified with likelihood 1-p or supplanting it out and out with likelihood ' $\mathrm{p}$ ". Limiting $\mathrm{n}(\mathrm{x}, \mathrm{y}) /$ to delivering just the extraordinary forces 0 or $z-1$ brings about salt-pepper clamour. The wellspring of drive clamour is generally the consequence of a mistake.

\subsection{Quantization Noise}

Quantization clamour is because of the quantisation of pixel esteems amid the simple to digital transformation. For instance, envision a simple picture with shine esteems going from 0 to 10 . On the off chance that was quantised to precision 0.1 the digitised picture will have 101 particular dim levels. A given force $\mathrm{z}$ could have initially been anyplace in the range. $\left(\mathrm{z}^{+}, .05\right)$ This vulnerability in the genuine estimation of $\mathrm{z}$ is called quantisation clamour.

\subsection{The Noise Function}

Generally, the properties of the commotion work $n(x, y)$ don't shift with $x$ andy. A spatially invariant stochastic process alluded to as being stationary. The commotion capacity could hypothetically take any frame, yet numerous standard likelihood conveyances have been discovered helpful. For added substance commotion, the Gaussian and Laplacian conveyances. The standard instance of motivation clamour utilises a uniform appropriation on $(0, \mathrm{z}-1)$. The most public clamour demonstrate utilised as a part of this postulation is an added substance zero-mean Gaussian of obscure difference, autonomously and indistinguishably disseminated for every pixel. The application to elective clamour models additionally considered. A few calculations created for added substance commotion can be adjusted to multiplicative clamour by logarithmically changing $f(x, y)$ applying the calculation, and afterwards applying the opposite change.

\section{Application of Digital Image Processing}

Computerized Image Processing connected in the fields of Computer vision, Face identification, 
Feature recognition, Lane takeoff cautioning framework, Non-photorealistic rendering, Medical picture preparing, Microscope picture handling Morphological picture handling, Remote detecting, and so on.

\subsection{PC Vision}

PC vision is the science and innovation of machines that see. As a logical train, PC vision is worried about the hypothesis for building counterfeit frameworks that get data from pictures. The picture information can take numerous structures, for example, a video grouping sees from various cameras, or multidimensional information from a therapeutic scanner[14].

As a mechanical teach, PC vision tries to apply its hypotheses and models to the development of PC vision frameworks. Cases of uses of PC vision incorporate frameworks for:

- Controlling procedures (e.g., a modern robot or an independent vehicle).

- Detecting occasions (e.g., for visual reconnaissance or individuals tallying).

- Organizing data (e.g., for ordering databases of pictures and picture successions).

- Modelling articles or conditions (e.g., mechanical examination, medicinal picture investigation or geographical demonstrating).

- Communication (e.g., as the contribution to a gadget for PC human connection).

Sub-spaces of PC vision incorporate scene recreation, occasion discovery, the video follows, and question acknowledgement, getting the hang of, ordering, movement estimation, and picture reclamation.

\subsection{Digital Video}

In electrical designing and software engineering, video handling is a specific instance of flag preparing, where the information and yield signals are video records or video streams. Video handling systems utilised as a part of TVs, VCRs, DVDs, video codecs, video players and different gadgets. For instance ordinarily, just outline and video preparing are distinctive in TV sets of various produces[15][16].

\subsection{Remote Sensing}

Remote detecting is the little or colossal scale obtaining of data of a protest or wonder, by the 
utilization of either recording or ongoing detecting device(s) that are remote, or not in physical or suggest contact with the question, (for example, by method for airship, shuttle, satellite, float, or ship). By and by, remote detecting is the standoff accumulation using an assortment of gadgets for social event data on a given question or region. Along these lines, Earth perception or climate satellite gathering stages, sea and barometrical watching climate float stages, the checking of a parolee by means of a ultrasound recognizable proof framework, Magnetic Resonance Imaging (MRI), Positron Emission Tomography (PET), X-radiation (X-RAY) and space tests are mostly cases of remote detecting. In present day utilisation, the term, by and large, alludes to the utilisation of imaging sensor innovations including instruments found in flying machine and rocket and also those utilised as a part of electrophysiology, and is unmistakable from other imaging-related fields, for example, medicinal imaging[15].

\section{Conclusion}

The picture handling requires a substantial measure of preparing power. In the disseminated condition where arrange, inactivity fundamentally influence the energy of execution the specific activities. There is requiring some security calculations in circulated picture preparing in customer server engineering. In the proposed work jpeg encoder and jpeg decoder will include for the elite with security in this design.

\section{References}

[1] M. Soni, A. Khare, S. Jain, A Survey of Digital Image Processing and its Problem, International Journal of Scientific and Research Publications, (2014), Vol.4, No.2, pp.1-6.

[2] D. Mukherjee, N. Ray, S. Acton, Level set analysis for leukocyte detection and tracking, IEEE Transactions on Image Processing, (2004), Vol.13, No.4, pp.562-572, DOI: 10.1109/TIP.2003.819858

[3] R. M. Nishikawa, K. L. Giger, K. Doi, C. J. Vyborny, R. A. Schmidt, Computer-aided detection of clustered microcalcifications on digital mammograms, Medical \& Biological Engineering \& Computing, (1995), Vol.33, No.2, pp.174-178, DOI: 10.1007/BF02523037

[4] S. Osher, J. A. Sethian, Fronts propagating with curvature dependent speed: Algorithms based on Hamilton Jacobi formulations, Journal of Computational Physics, (1988), Vol.79, No.1, pp.12-49, DOI: https://doi.org/10.1016/0021-9991(88)90002-2

[5] S. Aloni, Content-Based Image Retrieval in Biomedical Images Using SVM Classification with Relevance Feedback, International Journal of Scientific and Research Publications, (2013), Vol.3, No.11, pp.1-7. 
[6] J. Bouguet, Pyramidal implementation of the Lucas Kanade feature tracker Description of the algorithm, Intel Corporation Microprocessor Research Labs, (2000)

[7] E. D. Pisano, S. Zong, B. M. Hemminger, Contrast limited adaptive histogram equalisation image processing to improve the detection of simulated speculations in dense mammograms, Journal of Digital Imaging, (1998), Vol.11, No.4, pp.193-200, DOI: 10.1007/BF03178082

[8] A. A. Aly, S. B. Deris, N. Zaki, research review for digital image Segmentation techniques, International Journal of Computer Science \& Information Technology (IJCSIT), (2011), Vol.3, No.5, pp.99-106, DOI: 10.5121/ijcsit.2011.3509

[9] J. Smart, K. Hock, S. Csomor, Cross-Platform GUI Programming with wxWidgets, Prentice Hall, (2005)

[10] D. P. Mukherjee, N. Ray, S. T. Acton, Level set analysis for leukocyte detection and tracking, IEEE Transactions on Image Processing, (2004), Vol.13, No.4, pp.562-572.

[11] H. Coskun, Y. Li, M. A. Mackey, Ameboid cell motility: A model and inverse problem, with an application to live cell imaging data, Journal of Theoretical Biology, (2007), Vol.244, No.2, pp.169-179, DOI: $10.1016 /$ j.jtbi.2006.07.025

[12] M. Kass, A. Witkin, D. Terzopoulos, Snakes: Active contour models, International Journal of Computer Vision, (1987), Vol.1, pp.321-331, DOI: https://doi.org/10.1007/BF00133570

[13] K. Li, E. D. Miller, L. E. Weiss, P. G. Campbell, T. Kanade, Online tracking of migrating and proliferating cells imaged with phase - contrast microscopy, 2006 Conference on Computer Vision and Pattern Recognition Workshop (CVPR'06), (2006), June 17-22; New York, USA.

[14] P. Heinlein, J. Drexel, W. Schneider, Integrated Wavelets for Enhancement of Microcalcifications in Digital Mammography, IEEE Transactions on Medical Imaging, (2003), Vol.22, No.3, pp.402-413, DOI: 10.1109/TMI.2003.809632

[15] D. Sankar, T. Thomas, Fractal Modeling of Mammograms based on Mean and Variance for the Detection of Microcalcifications, International Conference on Computational Intelligence and Multimedia Applications, (2007), December 13-15; Sivakasi, India.

[16] M. Elter, C. Held, Semiautomatic segmentation for the computer-aided diagnosis of clustered microcalcifications, Proc. SPIE 6915, Medical Imaging 2008: Computer-Aided Diagnosis, 691524, (2008), February 16-21; San Diego, USA, DOI: https://doi.org/10.1117/12.770146 